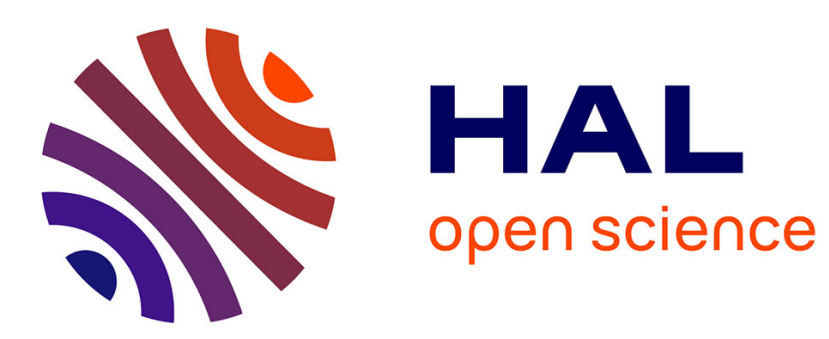

\title{
TP or not TP, that is the question
}

Robert Eymard, Thierry Gallouët, Cindy Guichard, Raphaèle Herbin, Roland

Masson

\section{To cite this version:}

Robert Eymard, Thierry Gallouët, Cindy Guichard, Raphaèle Herbin, Roland Masson. TP or not TP, that is the question. Computational Geosciences, 2014, pp.18:285-296, 2014. 10.1007/s10596013-9392-9 . hal-00801648v3

\section{HAL Id: hal-00801648 \\ https://hal.science/hal-00801648v3}

Submitted on 19 Jun 2013

HAL is a multi-disciplinary open access archive for the deposit and dissemination of scientific research documents, whether they are published or not. The documents may come from teaching and research institutions in France or abroad, or from public or private research centers.
L'archive ouverte pluridisciplinaire HAL, est destinée au dépôt et à la diffusion de documents scientifiques de niveau recherche, publiés ou non, émanant des établissements d'enseignement et de recherche français ou étrangers, des laboratoires publics ou privés. 


\title{
$\mathrm{TP}$ or not TP, that is the question
}

\author{
R. Eymard, T. Gallouët, C. Guichard, R. Herbin and R. Masson *
}

June 19, 2013

\begin{abstract}
We give here a comparative study of the mathematical analysis of two (classes of) discretisation schemes for the computation of approximate solutions to incompressible two phase flow problem in homogeneous porous media. The first scheme is the well-known finite volume scheme with a two-point flux approximation, classically used in industry. The second class contains the so-called approximate gradient schemes, which include finite elements with mass lumping, mixed finite elements, mimetic finite differences. Both (classes of) schemes are nonconforming and can be expressed using discrete function and gradient reconstructions within a variational formulation. Each class has its specific advantages and drawbacks: monotony properties are natural with the two point finite volume scheme, but meshes are restricted due to consistency issues; on the contrary, gradient schemes can be used on general meshes, but monotony properties are difficult to obtain.
\end{abstract}

KEYwORDS. two-phase flow in porous media, two-point flux approximation, finite volume scheme, gradient scheme.

\section{Introduction}

Ideally, discretisation schemes should be both consistent and robust. By consistent we mean that if the scheme converges, then it converges to a (weak) solution of the problem under study, and by robust, we mean that it preserves the physical properties of the unknowns. In the past several decades, a great deal of effort has been put into finding this ideal scheme for the simulation of fluid flow in porous media, which would be both consistent on any mesh and robust: we refer to e.g $[13,18]$ and references therein for a battery of tests and schemes for anisotropic and heteregenous problems.

Here we concentrate on the finite volume scheme with two point flux approximation, which is widely used in industry, and which we shall call the TP scheme here for short, and on the class of gradient schemes, which can be shown to include well-known schemes such as the conforming finite elements with mass lumping, also known as Control Volume Finite Element, mixed finite elements, and some MPFA schemes, as well as some recently developed schemes such as the mimetic method, the mixed and hybrid finite volumes or the SUSHI scheme [12]. Our aim here is to analyze the mathematical properties of these two (classes of) schemes, in order to better understand their mechanisms. To this purpose, we choose to work on a simplified model for two-phase flow in porous media problem, which is clearly more

* Robert Eymard: L.A.M.A., UMR 8050, Université Paris-Est robert. eymardeuniv-mlv . fr Thierry Gallouët, Raphaèle Herbin: LATP, UMR 7353, Aix-Marseille Université

thierry.gallouet, raphaele.herbin@univ-amu.fr

Cindy Guichard, Roland Masson: L.J.A.D., UMR 7351, Université de Nice Sophia Antipolis \& team Coffee INRIA Sophia Antipolis Méditerranée, cindy.guichard, roland.masson@unice.fr 
difficult than a mere diffusion problem but which remains simple enough to compare the different steps in the mathematical analysis.

Let $\Omega$ be an open bounded subset of $\mathbb{R}^{d}(d \geq 0)$, let $T \in \mathbb{R}_{+}$. The saturation $\left.u: \Omega \times\right] 0, T[\rightarrow \mathbb{R}$ and the pressures $\left.p_{1}, p_{2}: \Omega \times\right] 0, T[\rightarrow \mathbb{R}$ of the two phases are solution to the following coupled system posed on the domain $\Omega \times] 0, T[$ :

$$
\begin{aligned}
u_{t}-\operatorname{div}\left(k_{1}(u) \nabla p_{1}\right) & =f_{1}(c) s^{+}-f_{1}(u) s^{-} \\
(1-u)_{t}-\operatorname{div}\left(k_{2}(u) \nabla p_{2}\right) & =f_{2}(c) s^{+}-f_{2}(u) s^{-} \\
p_{2}-p_{1} & =p_{c}(u)
\end{aligned}
$$

The functions $k_{1}(u)$ and $k_{2}(u)$ respectively denote the mobilities of the wetting fluid and of the nonwetting fluid and the function $p_{c}(u)$ represents the capillary pressure. The functions $s^{+}$and $s^{-}$stand respectively for an injection and a production volumetric flow rate. The composition of the injected fluid in the wetting and non wetting components is prescribed by the imposed input saturation $c$, whereas that of the produced fluid depends on the saturation $u$, by means of "the fractional flows" $f_{1}$ and $f_{2}$ of the wetting and non-wetting phases defined by:

$$
\begin{aligned}
& f_{1}(a)=\frac{k_{1}(a)}{k_{1}(a)+k_{2}(a)}, \\
& f_{2}(a)=\frac{k_{2}(a)}{k_{1}(a)+k_{2}(a)}=1-f_{1}(a),
\end{aligned} \quad \forall a \in[0,1] .
$$

Following Chavent [6], in order to obtain a weak formulation (which is shown in the sequel to be the limit of the numerical scheme), we introduce the following primitive functions $\tilde{p}_{1}$ and $\tilde{p}_{2}$ :

$$
\begin{array}{r}
\tilde{p}_{1}(b)=\int_{1}^{b} \frac{k_{2}(a)}{k_{1}(a)+k_{2}(a)} p_{c}{ }^{\prime}(a) \mathrm{d} a \text { and } \\
\tilde{p}_{2}(b)=\int_{1}^{b} \frac{k_{1}(a)}{k_{1}(a)+k_{2}(a)} p_{c}{ }^{\prime}(a) \mathrm{d} a, \forall b \in[0,1] .
\end{array}
$$

The "global pressure" is then defined as $p=p_{1}+\tilde{p}_{1}(u)=p_{2}-\tilde{p}_{2}(u)$ (indeed we have $p_{2}-p_{1}=$ $p_{c}(u)=\tilde{p}_{1}(u)+\tilde{p}_{2}(u)$ ). Let us finally define the function $\psi$ by:

$$
\psi(b)=-\int_{0}^{b} \frac{k_{1}(a) k_{2}(a)}{k_{1}(a)+k_{2}(a)} p_{c}{ }^{\prime}(a) d a, \forall b \in[0,1] .
$$

We then deduce that the saturation $u$ and the global pressure $p$ are solutions to the following system, posed on the domain $\Omega \times] 0, T[$ :

$$
\begin{aligned}
u_{t}+\operatorname{div}\left(f_{1}(u) \boldsymbol{v}-\nabla \psi(u)\right) & =f_{1}(c) s^{+}-f_{1}(u) s^{-}, \\
\operatorname{div}(\boldsymbol{v}) & =s^{+}-s^{-} \\
\boldsymbol{v} & =-M(u) \nabla p
\end{aligned}
$$

where $M(u)=k_{1}(u)+k_{2}(u)$ is the total mobility. Since our aim is to compare the main properties of discretisation schemes, we assume homogeneous Dirichlet boundary conditions on $p$ and $\psi(u)$ :

$$
\begin{aligned}
\psi(u) & =0 \text { on } \partial \Omega \times] 0, T[, \\
p & =0 \text { on } \partial \Omega \times] 0, T[.
\end{aligned}
$$


We complete the strong formulation of the problem with the following initial condition:

$$
u(\cdot, 0)=u_{\text {ini }} \text { on } \Omega .
$$

Then the following conservation equation for the second phase is a consequence of (6)-(8) (which shows that the two phases are treated symmetrically):

$$
(1-u)_{t}+\operatorname{div}\left(f_{2}(u) \boldsymbol{v}+\nabla \psi(u)\right)=f_{2}(c) s^{+}-f_{2}(u) s^{-} .
$$

The data are assumed to satisfy the following assumptions:

- $\Omega$ is a polygonal connected subset of $\mathbb{R}^{d}, d=1,2$ or 3 , and $T>0$ is given,

- $u_{\text {ini }} \in L^{\infty}(\Omega)$, and $0 \leq u_{\text {ini }} \leq 1$ a.e in $\Omega$,

- $c \in L^{\infty}(\Omega)$, and $0 \leq c \leq 1$ a.e in $\Omega$,

- $s^{+}, s^{-} \in L^{2}(\Omega), s^{+}$and $s^{-} \geq 0$ a.e.in $\Omega$,

- $f_{1}$ is a non-decreasingcontinuous function from $\mathbb{R}$ to $[0,1]$ s.t.

$$
\begin{aligned}
& f_{1}(x)=f_{1}(0)=0, \text { for all } x \in(-\infty, 0], \\
& f_{1}(x)=f_{1}(1)=1, \text { for all } x \in[1,+\infty),
\end{aligned}
$$

- $M$ is a nonnegative continuous function from $\mathbb{R}$ to $\mathbb{R}$ and there exist real values $0<\underline{M} \leq \bar{M}$ s.t. $\underline{M} \leq M(x) \leq \bar{M}$ for all $x \in \mathbb{R}$,

- $\psi$ is (strictly) increasing and Lipschitz-continuous

from $\mathbb{R}$ to $\mathbb{R}$ with constant $L_{\psi}$ s.t. $\psi(0)=0$,

$|\psi(x)| \geq A|x|-B$ for all $x \in \mathbb{R}$, for some given values $A, B \in(0,+\infty)$.

Let us emphasize that these hypotheses correspond to industrial situations. In particular, the fact that the function $\psi$ is assumed to be Lipschitz continuous corresponds to the fact that the functions $k_{1}$ and $k_{2}$ tend to zero faster than the possible singularity of the capillary pressure function.

Note that functions $M, f_{1}$ and $\psi$ are assumed to be defined on $\mathbb{R}$, even though they are functions of the saturation; indeed, we are not able to prove that the approximation of the saturation remains in $[0,1]$ when using a gradient scheme (see Section 3).

Definition 1.1 (Weak solution) Under assumptions and definitions (12), the pair $(u, p)$ is a weak solution of Problem (6)-(11) if

$$
\begin{aligned}
& u \in L^{2}(\Omega \times] 0, T[), \\
& p \in L^{2}\left(0, T ; H_{0}^{1}(\Omega)\right), \\
& \psi(u) \in L^{2}\left(0, T ; H_{0}^{1}(\Omega)\right),
\end{aligned}
$$


and for every function $\varphi \in C_{c}^{\infty}(\Omega \times[0, T[)$,

$$
\begin{aligned}
& \int_{0}^{T} \int_{\Omega}\left[-u \varphi_{t}-\left(f_{1}(u) \boldsymbol{v}-\nabla \psi(u)\right) \cdot \nabla \varphi\right] \mathrm{d} \boldsymbol{x} \mathrm{d} t-\int_{\Omega} u_{\text {ini }} \varphi(., 0) \mathrm{d} \boldsymbol{x} \\
& \quad=\int_{0}^{T} \int_{\Omega}\left(f_{1}(c) s^{+}-f_{1}(u) s^{-}\right) \varphi \mathrm{d} \boldsymbol{x} \mathrm{d} t \\
& \int_{0}^{T} \int_{\Omega}\left[\boldsymbol{v} \cdot \nabla \varphi+\left(s^{+}-s^{-}\right) \varphi\right] \mathrm{d} \boldsymbol{x} \mathrm{d} t=0, \\
& \boldsymbol{v}=-M(u) \nabla p \text { a.e. in } \Omega \times] 0, T[,
\end{aligned}
$$

where we denote by $C_{c}^{\infty}\left(\Omega \times\left[0, T[)\right.\right.$ the set of the restrictions of functions of $C_{c}^{\infty}(\Omega \times]-\infty, T[)$ to $\Omega \times[0, T[$.

The remainder of this paper is concerned with the discretisation of Problem (6)-(11). The TP method is presented in Section 2, and the gradient scheme is described in Section 3. Some conclusions and perspectives are drawn in Section 4.

\section{Approximation by the TP finite volume scheme}

\subsection{The mesh and the discrete operators}

The Two-Point Flux Approximation (TPFA in the literature, TP for short) is used to discretise diffusion terms under the form $-\operatorname{div} \lambda \nabla u$ (a few terms under this form occur in Problem (6)-(11)) on a finite volume mesh $\mathcal{M}$ satisfying the following orthogonality condition: each control volume $K \in \mathcal{M}$ is assumed to contain a point $\boldsymbol{x}_{K}$ such that, for each pair of neighboring control volumes $K, L \in \mathcal{M}$, the line $\left(\boldsymbol{x}_{K}, \boldsymbol{x}_{L}\right)$ is orthogonal to the common interface $K \mid L$ (see Fig.1). This leads to a strong restriction on the meshes; main examples of such meshes are rectangular parallelepipedic boxes in 3D (possibly distorted in the case of the so-called "corner point geometry") or Voronoï boxes (in which case the control volume relative to $\boldsymbol{x}_{K}$ are all the points that are closer to $\boldsymbol{x}_{K}$ than to any other center point.). For a given $K \in \mathcal{M}$, we denote by $\mathcal{E}_{K}$ the set of all the faces of $K$, and we define the set of all faces

$$
\mathcal{E}=\bigcup_{K \in \mathcal{M}} \mathcal{E}_{K}
$$

For any $\sigma \in \mathcal{E}$, we define (see Fig.1) the set $D_{\sigma} \subset \Omega$ by:

- the union of the two cones with basis $\sigma$ and respective vertices $\boldsymbol{x}_{K}$ and $\boldsymbol{x}_{L}$ if $\sigma$ is the interface between control volumes $K$ and $L$,

- the cone with basis $\sigma$ and vertex $\boldsymbol{x}_{K}$ if $\sigma$ is a boundary face and a face of the control volume $K$.

We denote by $\boldsymbol{n}_{K, \sigma}$ the unit vector, normal to $\sigma$, oriented outward to $K$. For any face $\sigma$, we define $d_{\sigma}$ as the distance between $\boldsymbol{x}_{K}$ and $\boldsymbol{x}_{L}$ if $\sigma$ is the interface between control volumes $K$ and $L$, and as the orthogonal distance between $\boldsymbol{x}_{K}$ and $\sigma$ if $\sigma \in \mathcal{E}_{K}$ is included in the boundary of the domain. 


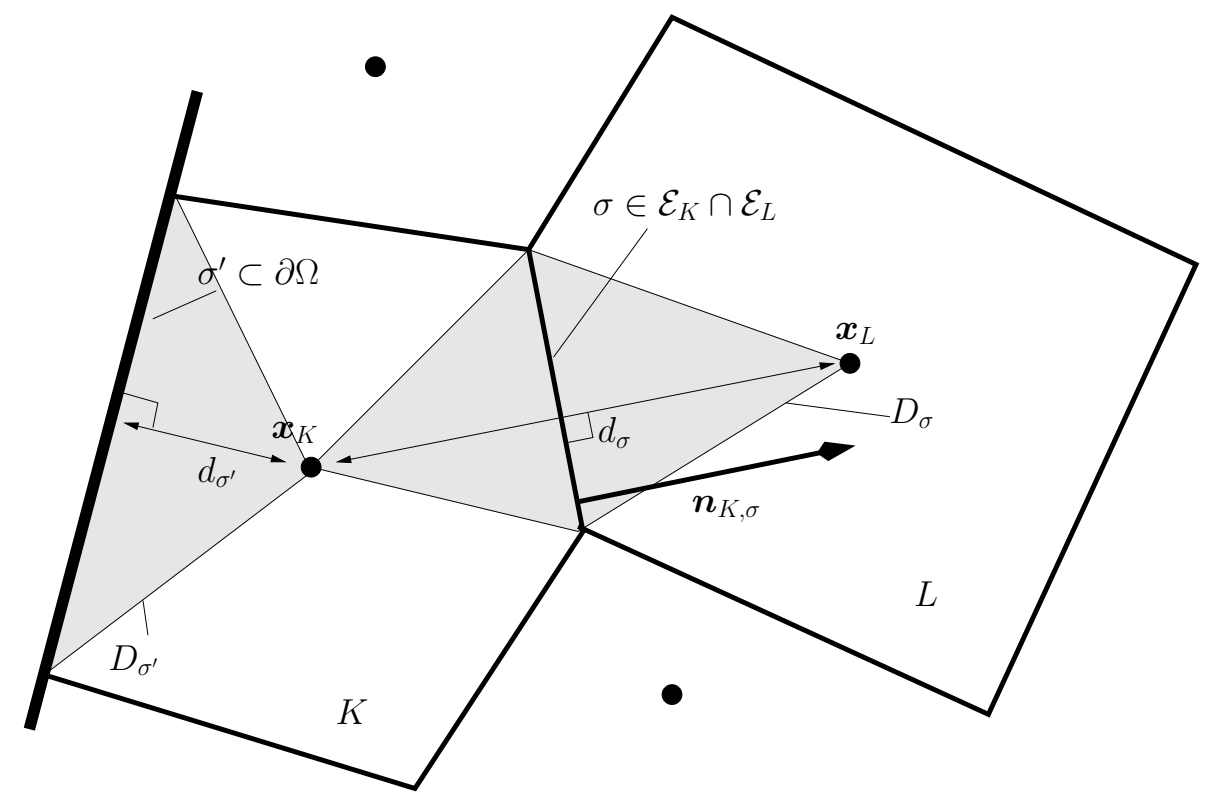

Figure 1: Two control volumes of an admissible mesh

We then denote by $X_{\mathcal{D}, 0}=\mathbb{R}^{\mathcal{M}}$, and for $u \in X_{\mathcal{D}, 0}$, we denote by $\Pi_{\mathcal{D}} u \in L^{2}(\Omega)$ the piecewise constant function equal to $u_{K}$ in $K \in \mathcal{M}$. Then for any $u \in X_{\mathcal{D}, 0}$ and $\sigma \in \mathcal{E}_{K}$, we let $u_{K, \sigma}=u_{L}$ if $\sigma$ is the interface between control volumes $K$ and $L$, and $u_{K, \sigma}=0$ if $\sigma$ is a boundary face. The finite volume method consists in first writing

$$
-\int_{K} \operatorname{div}(\lambda \nabla u) \mathrm{d} \boldsymbol{x}=-\sum_{\sigma \in \mathcal{E}_{K}} \int_{\sigma} \lambda \nabla u \cdot \boldsymbol{n}_{K, \sigma} \mathrm{d} s,
$$

and then specifying the approximation $F_{K, \sigma}$ of the flux $-\int_{\sigma} \lambda \nabla u \cdot \boldsymbol{n}_{K, \sigma} \mathrm{d} s$. The TP numerical flux $F_{K, \sigma}$ is defined by:

$$
F_{K, \sigma}=-\lambda_{\sigma} \frac{|\sigma|}{d_{\sigma}}\left(u_{K, \sigma}-u_{K}\right), \forall \sigma \in \mathcal{E}_{K},
$$

where $|\sigma|$ is the area of $\sigma$ in the 3D case (length in the 2D case and 1 in the $1 \mathrm{D}$ case), and $\lambda_{\sigma}$ is a suitable approximation of $\lambda$ on $\sigma$.

The numerical flux is then consistent under the orthogonality assumption on the mesh (and this is actually the reason why we need this assumption) in the sense that, for any function $\varphi \in C_{c}^{\infty}(\Omega)$,

$$
\left|\int_{\sigma} \nabla \varphi \cdot \boldsymbol{n}_{K, \sigma}-\frac{|\sigma|}{d_{\sigma}}\left(v_{K, \sigma}-v_{K}\right)\right| \leq|\sigma| C_{\varphi} h
$$

where $v \in X_{\mathcal{D}, 0}$ is defined by $v_{K}=\varphi\left(\boldsymbol{x}_{K}\right)$ for all $K \in \mathcal{M}, C_{\varphi} \in \mathbb{R}_{+}$depends only on $\varphi$ and $h=\max \{\operatorname{diam}(K), K \in \mathcal{M}\}$ is the size of the mesh. This consistency property is crucial in order for the scheme to converge to the correct solution.

Note that the important following local conservation property, characterizing the finite volume framework, holds in the case where $\sigma$ is the interface between the control volumes $K$ and $L$ :

$$
F_{K, \sigma}+F_{L, \sigma}=0 \text {. }
$$


We may write the TP finite volume schemes with discrete gradient operators. To this purpose, for any $u \in X_{\mathcal{D}, 0}$, we define the piecewise constant function $\widehat{\nabla}_{\mathcal{D}} u \in L^{2}(\Omega)^{d}$ whose constant value $\widehat{\nabla}_{\sigma} u$ on $D_{\sigma}$ is defined by:

$$
\widehat{\nabla}_{\sigma} u=d \frac{u_{K, \sigma}-u_{K}}{d_{\sigma}} \boldsymbol{n}_{K, \sigma}, \forall K \in \mathcal{M}, \forall \sigma \in \mathcal{E}_{K}
$$

(Recall that $d$ is the space dimension.) We also define, for any $v \in X_{\mathcal{D}, 0}$, a piecewise constant function $\widetilde{\nabla}_{\mathcal{D}} v \in L^{2}(\Omega)^{d}$ whose constant value $\widetilde{\nabla}_{\sigma} v$ on $D_{\sigma}$ is such that:

$$
\widetilde{\nabla}_{\sigma} v \cdot \boldsymbol{n}_{K, \sigma}=\frac{v_{K, \sigma}-v_{K}}{d_{\sigma}}, \forall K \in \mathcal{M}, \forall \sigma \in \mathcal{E}_{K} .
$$

The discrete normal gradient thus defined is consistent with the normal gradient: this is in fact the same property as the conservativity of the flux (17), obtained thanks to the orthogonality assumption on the mesh. We complete the definition of $\widetilde{\nabla}_{\sigma} v$ by any consistent reconstruction in the hyperplane parallel to $\sigma$, chosen such that if $\varphi \in C_{c}^{\infty}(\Omega)$ and $v \in X_{\mathcal{D}, 0}$ is defined by $v_{K}=\varphi\left(\boldsymbol{x}_{K}\right)$ for all $K \in \mathcal{M}$, then $\widetilde{\nabla}_{\mathcal{D}} v$ converges in $L^{2}(\Omega)^{d}$ to $\nabla \varphi$ as the space step tends to 0 : the whole discrete gradient $\widetilde{\nabla}$ is therefore consistent.

If we now define by $\lambda_{\mathcal{D}}$ the piecewise constant function with value $\lambda_{\sigma}$ on $D_{\sigma}$, and using $\left|D_{\sigma}\right|=\frac{1}{d}|\sigma| d_{\sigma}$, we infer from (16) that

$$
\begin{aligned}
\sum_{K \in \mathcal{M}} v_{K} & \sum_{\sigma \in \mathcal{E}_{K}} F_{K, \sigma}=\int_{\Omega} \lambda_{\mathcal{D}} \widehat{\nabla}_{\mathcal{D}} u \cdot \widetilde{\nabla}_{\mathcal{D}} v \mathrm{~d} \boldsymbol{x} \\
& =\sum_{\sigma \in \mathcal{E}_{K} \cap \mathcal{E}_{L}} \lambda_{\sigma} \frac{|\sigma|}{d_{\sigma}}\left(u_{L}-u_{K}\right)\left(v_{L}-v_{K}\right)+\sum_{\sigma \in \mathcal{E}_{K}, \sigma \subset \partial \Omega} \lambda_{\sigma} \frac{|\sigma|}{d_{\sigma}} u_{K} v_{K}
\end{aligned}
$$

which leads to

$$
\int_{\Omega} \lambda_{\mathcal{D}} \widehat{\nabla}_{\mathcal{D}} u \cdot \widetilde{\nabla}_{\mathcal{D}} u \mathrm{~d} \boldsymbol{x}=\frac{1}{d} \int_{\Omega} \lambda_{\mathcal{D}}\left|\widehat{\nabla}_{\mathcal{D}} u\right|^{2} \mathrm{~d} \boldsymbol{x}
$$

\subsection{The TP scheme for the two-phase flow problem}

Let us consider the TP scheme for the discretisation of Problem (6)-(11). We consider a finite volume discretisation $\mathcal{D}$ following the specification of the preceding section, and a discrete time sequence $t^{(0)}=$ $0<t^{(1)} \ldots<t^{(N)}=T$; the time step is defined as $\delta t^{\left(n+\frac{1}{2}\right)}=t^{(n+1)}-t^{(n)}$ for $n=0, \ldots, N-1$, and the discrete initial condition $u_{K}^{(0)}$ on cell $K$ as the average value of $u_{\text {ini }}$ on $K$. Then, for $n=0, \ldots, N-1$, we look for $\left(u^{(n+1)}, p^{(n+1)}\right) \in X_{\mathcal{D}, 0}^{2}$ such that

$$
\begin{aligned}
& \frac{u_{K}^{(n+1)}-u_{K}^{(n)}}{\delta t^{\left(n+\frac{1}{2}\right)}}|K|+ \\
& \sum_{\sigma \in \mathcal{E}_{K}}|\sigma|\left(\left(V_{K, \sigma}^{(n+1)}\right)^{+} f_{1}\left(u_{K}^{(n+1)}\right)-\left(V_{K, \sigma}^{(n+1)}\right)^{-} f_{1}\left(u_{K, \sigma}^{(n+1)}\right)-\frac{\psi\left(u_{K, \sigma}^{(n+1)}\right)-\psi\left(u_{K}^{(n+1)}\right)}{d_{\sigma}}\right) \\
& =f_{1}\left(c_{K}\right) s_{K}^{+}-f_{1}\left(u_{K}^{(n+1)}\right) s_{K}^{-}, \\
& \sum_{\sigma \in \mathcal{E}_{K}}|\sigma| V_{K, \sigma}^{(n+1)}=s_{K}^{+}-s_{K}^{-}, \\
& \text {with } V_{K, \sigma}^{(n+1)}=-M_{\sigma}^{(n+1)} \frac{p_{K, \sigma}^{(n+1)}-p_{K}^{(n+1)}}{d_{\sigma}}
\end{aligned}
$$


where $s_{K}^{ \pm}=\int_{K} s^{ \pm}(\boldsymbol{x}) \mathrm{d} \boldsymbol{x}, c_{K}$ is the average value of $c$ in $K, M_{\sigma}^{(n+1)}$ is equal to $M(0)$ for an exterior edge, and any averaging value between $M\left(u_{K}^{(n+1)}\right)$ and $M\left(u_{L}^{(n+1)}\right)$ in the case where $\sigma$ is the interface between control volumes $K$ and $L$. Note that the above scheme is upstream weighted with respect to the convection term in (20).

One may rewrite Scheme (20)-(21), introducing as in (18) the above defined discrete gradient operators. This formulation of the TP scheme has a few common points with (27) corresponding to the gradient scheme presented in Section 3.

$$
\begin{aligned}
& u^{(0)} \in X_{\mathcal{D}, 0}, u^{(n+1)} \in X_{\mathcal{D}, 0}, p^{(n+1)} \in X_{\mathcal{D}, 0}, \\
& \delta_{\mathcal{D}}^{\left(n+\frac{1}{2}\right)} u=\Pi_{\mathcal{D}} \frac{u^{(n+1)}-u^{(n)}}{\delta t^{\left(n+\frac{1}{2}\right)}}, \\
& \int_{\Omega}\left(\delta_{\mathcal{D}}^{\left(n+\frac{1}{2}\right)} u \Pi_{\mathcal{D}} w-\left(f_{1}\left(\Pi_{\mathcal{D}}^{\mathrm{up}} u^{(n+1)}\right) \boldsymbol{v}_{\mathcal{D}}^{(n+1)}-\widehat{\nabla}_{\mathcal{D}} \psi\left(u^{(n+1)}\right)\right) \cdot \widetilde{\nabla}_{\mathcal{D}} w\right) \mathrm{d} \boldsymbol{x} \\
& =\int_{\Omega}\left(f_{1}\left(c_{\mathcal{D}}\right) s^{+}-f_{1}\left(\Pi_{\mathcal{D}} u^{(n+1)}\right) s^{-}\right) \Pi_{\mathcal{D}} w \mathrm{~d} \boldsymbol{x}, \\
& -\int_{\Omega} \boldsymbol{v}_{\mathcal{D}}^{(n+1)} \cdot \widetilde{\nabla}_{\mathcal{D}} w \mathrm{~d} \boldsymbol{x}=\int_{\Omega}\left(s^{+}-s^{-}\right) \Pi_{\mathcal{D}} w \mathrm{~d} \boldsymbol{x}, \\
& \boldsymbol{v}_{\mathcal{D}}^{(n+1)}=-M\left(u^{(n+1)}\right) \widehat{\nabla}_{\mathcal{D}} p^{(n+1)}, \\
& \forall w \in X_{\mathcal{D}, 0}, \forall n=0, \ldots, N-1,
\end{aligned}
$$

where $\Pi_{\mathcal{D}}^{\text {up }} u^{(n+1)}$ is the upstream value of $u^{(n+1)}$ on each $D_{\sigma}$, the piecewise constant function $c_{\mathcal{D}}$ is equal to $c_{K}$ on each control volume $K, M\left(u^{(n+1)}\right)$ is equal to the average value $M_{\sigma}^{(n+1)}$ on each diamond cell $D_{\sigma}$ (see Fig.1), and with the same notations for the definition of space-time dependent functions, that is, for any $t \in\left(t^{(n)}, t^{(n+1)}\right], n=0, \ldots, N-1$ and for a.e. $\boldsymbol{x} \in \Omega$ :

$$
\begin{aligned}
& \Pi_{\mathcal{D}} u(\boldsymbol{x}, 0)=\Pi_{\mathcal{D}} u^{(0)}(\boldsymbol{x}), \\
& \Pi_{\mathcal{D}} u(\boldsymbol{x}, t)=\Pi_{\mathcal{D}} u^{(n+1)}(\boldsymbol{x}), \\
& \Pi_{\mathcal{D}} \psi(u)(\boldsymbol{x}, t)=\Pi_{\mathcal{D}} \psi\left(u^{(n+1)}\right)(\boldsymbol{x}), \\
& \Pi_{\mathcal{D}}^{\mathrm{up}} u(\boldsymbol{x}, t)=\Pi_{\mathcal{D}}^{\mathrm{up}} u^{(n+1)}(\boldsymbol{x}), \\
& \widehat{\nabla}_{\mathcal{D}} \psi(u)(\boldsymbol{x}, t)=\widehat{\nabla}_{\mathcal{D}} \psi\left(u^{(n+1)}\right)(\boldsymbol{x}), \\
& \Pi_{\mathcal{D}} p(\boldsymbol{x}, t)=\Pi_{\mathcal{D}} p^{(n+1)}(\boldsymbol{x}), \\
& \widehat{\nabla}_{\mathcal{D}} p(\boldsymbol{x}, t)=\widehat{\nabla}_{\mathcal{D}} p^{(n+1)}(\boldsymbol{x}) .
\end{aligned}
$$

An important difference between (22)-(23) and (27) is that there are two discrete gradients defined for an element of $X_{\mathcal{D}, 0}$. The first one is applied to the unknown fields $\left(\widehat{\nabla}_{\mathcal{D}}\right)$, and converges only weakly; the second one $\left(\widetilde{\nabla}_{\mathcal{D}}\right)$ is applied to the test functions and converges strongly. In the framework of the gradient schemes presented in Section 3, only one discrete gradient is used: this has been the main difficulty in 
going from the TP finite volume scheme to the gradient schemes: indeed, this gradient must be such that we can get some estimates in a discrete $H^{1}$ norm (as the first gradient $\widehat{\nabla}_{\mathcal{D}}$ ) but also be consistent, as the second gradient $\widetilde{\nabla}_{\mathcal{D}}$.

\subsection{Convergence analysis}

The convergence proof of Scheme (20)-(21) is detailed in [21] (in the case of Neumann boundary conditions instead of Dirichlet ones). Let us sketch its principles; some are close to those presented in Section 3; we stress those which are specific to the TP framework. Consider a sequence of finite volume discretisations with space and time steps tending to 0 .

1. Let us first show that any solution $\left(u^{(n+1)}, p^{(n+1)}\right) \in X_{\mathcal{D}, 0}^{2}$ is such that $0 \leq u_{K}^{(n+1)} \leq 1$ for all $K \in \mathcal{M}$ and $n=0, \ldots, N-1$. Multiply (21) by $f_{1}\left(u_{K}^{(n+1)}\right)$ and subtract to (20); let $\bar{K} \in \mathcal{M}$ realizing the maximum (resp. minimum) value of the family $\left(u_{K}^{(n+1)}\right)_{K \in \mathcal{M}, n=0, \ldots, N-1}$, assumed to be greater than 1 (resp. negative).

Then $f_{1}\left(u_{\bar{K}}^{(n+1)}\right)-f_{1}\left(u_{\bar{K}, \sigma}^{(n+1)}\right)$ is non negative (resp. non positive), and a contradiction follows, thanks to hypotheses $(12 b),(12 c)$. Note that this estimate is not in general possible for gradient schemes.

2. The existence of a solution is then obtained from this estimate, thanks to a standard topological degree argument (this is also the case for gradient schemes).

3. Following the lines of [10, chapter 4], letting $w=p^{(n+1)}$ in (23), we get, for the same reason as in (19), an estimate on the norm of $\widehat{\nabla}_{\mathcal{D}} p$ in $L^{2}(\Omega \times] 0, T[)^{d}$. This leads to the existence of a function $\bar{p} \in L^{2}\left(0, T ; H_{0}^{1}(\Omega)\right)$ such that, up to a subsequence, $\Pi_{\mathcal{D}} p$ (resp. $\widehat{\nabla}_{\mathcal{D}} p$ ) weakly converges to $\bar{p}$ (resp. $\nabla \bar{p})$ in $L^{2}(\Omega \times] 0, T\left[\right.$ ) (resp. $\left.L^{2}(\Omega \times] 0, T[)^{d}\right)$. This step is quite similar to the proof of (32) in Section 3.

4. Similarly to [21], we let $w=\delta t^{\left(n+\frac{1}{2}\right)} u^{(n+1)}$ in (22), and we sum on $n=0, \ldots, N-1$. We then slightly improve the proof of [21] by introducing, as in [11], the primitive $\zeta$ of $\sqrt{\psi^{\prime}}$ and by using the relation

$$
(\zeta(a)-\zeta(b))^{2} \leq(a-b)(\psi(a)-\psi(b)), \forall(a, b) \in \mathbb{R}^{2} .
$$

We then get an estimate on the norm of $\widehat{\nabla}_{\mathcal{D}} \zeta(u)$ in $L^{2}(\Omega \times] 0, T[)^{d}$; note that this estimate is obtained thanks to the positivity of the transmissivities in the approximate diffusion operator when using the TP scheme (more on this in the discussion). It is not possible to obtain such an estimate in this way for a general gradient scheme. This uniform $L^{2}$ estimate on $\widehat{\nabla}_{\mathcal{D}} \zeta(u)$ yields the existence of $\bar{\zeta} \in L^{2}\left(0, T ; H_{0}^{1}(\Omega)\right)$ such that (up to a subsequence) $\Pi_{\mathcal{D}} \zeta(u)$ (resp. $\widehat{\nabla}_{\mathcal{D}} \zeta(u)$ ) weakly converges to $\bar{\zeta}$ (resp. $\nabla \bar{\zeta})$ in $L^{2}(\Omega \times] 0, T\left[\right.$ ) (resp. $\left.L^{2}(\Omega \times] 0, T[)^{d}\right)$. Note that the convective term poses no problem in the obtention of this estimate: thanks to the upstream weighting scheme, it yields a positive term; in fact, this positive term leads to the so-called weak BV inequality, which is needed in the case where $\psi$ is only assumed to be non decreasing (case of degenerate diffusion problems [11]). This step highly differs from the proof of (33) in Section 3.

5. The Alt\&Luckhaus technique [2] allows to get a time translate estimate on $\zeta(u)$, and therefore strong convergence to $\bar{\zeta}$. From an estimate on the norm of $\widehat{\nabla}_{\mathcal{D}} \zeta(u)$ in $L^{2}(\Omega \times] 0, T[)^{d}[10]$, we get an estimate on the space translates, and therefore, $\Pi_{\mathcal{D}} \zeta(u)$ converges to $\bar{\zeta}$ in $L^{2}(\Omega \times] 0, T[$ ) (up 
to a subsequence), and $\Pi_{\mathcal{D}} u$ also converges to $\bar{u}=\zeta^{(-1)}(\bar{\zeta})$ in $L^{2}(\Omega \times] 0, T[)$. This step is quite similar to Lemma 3.2 in Section 3.

We then get that $\Pi_{\mathcal{D}}^{\text {up }} u$ (resp. $M(u)$ ) converge to $\bar{u}($ resp. $M(\bar{u}))$ in $L^{2}(\Omega \times] 0, T[$ ), thanks to the estimate on the norm of $\widehat{\nabla}_{\mathcal{D}} \zeta(u)$ in $L^{2}(\Omega \times] 0, T[)^{d}$. This step results from the use of upstream weighting and averaging in the TP method.

6. Defining, for any regular function $\varphi \in C_{c}^{\infty}\left(\Omega \times\left[0, T[)\right.\right.$, the interpolation $v^{(n)} \in X_{\mathcal{D}, 0}$ by $v_{K}^{(n)}=$ $\varphi\left(\boldsymbol{x}_{K}, t^{(n)}\right)$ for all $K \in \mathcal{M}$, we consider in Scheme (22),(23) the test function $\delta t^{\left(n+\frac{1}{2}\right)} v^{(n)}$ and sum on $n=0, \ldots, N-1$. We may then pass to the limit (thanks to the consistency of $\widetilde{\nabla}_{\mathcal{D}}$ ), and we get that the pair $(\bar{u}, \bar{p})$ is a weak solution of the problem, in the sense of Definition 1.1. This step follows some common ideas with Lemma 3.4 in Section 3.

\section{Approximation by a gradient scheme}

We now turn to the approximation of Problem (6)-(11) by an approximate gradient scheme. We recall that the family of approximate gradient schemes provides a generic framework for the approximation of various linear or nonlinear problems [7,9].

\subsection{The mesh and the discrete operators}

We present here the framework of gradient discretisations for diffusion problems with homogeneous Dirichlet boundary conditions. This framework enables us to consider schemes applying on general meshes (neither orthogonality nor conformity conditions are needed).

\section{Definition 3.1 (Gradient discretisation for homogeneous Dirichlet problems)}

A gradient discretisation for homogeneous Dirichlet problems $\mathcal{D}$ is defined by $\mathcal{D}=\left(X_{\mathcal{D}, 0}, \Pi_{\mathcal{D}}, \nabla_{\mathcal{D}}\right)$, where:

1. the set $X_{\mathcal{D}, 0}$ of discrete unknowns is a finite dimensional vector space on $\mathbb{R}$,

2. the linear mapping $\Pi_{\mathcal{D}}: X_{\mathcal{D}, 0} \rightarrow L^{2}(\Omega)$ is the reconstruction of the approximate function,

3. the linear mapping $\nabla_{\mathcal{D}}: X_{\mathcal{D}, 0} \rightarrow L^{2}(\Omega)^{d}$ is the discrete gradient operator.

The discrete gradient operator $\nabla_{\mathcal{D}}$ must be chosen such that $\|\cdot\|_{\mathcal{D}}$ defined by $\|w\|_{\mathcal{D}}=\left\|\nabla_{\mathcal{D}} w\right\|_{L^{2}(\Omega)^{d}}$ is a norm on $X_{\mathcal{D}, 0}$.

The associated gradient scheme is the application of these operators to a given (diffusion) problem. Our aim is to prove the convergence of an approximate gradient scheme for Problem (6)-(11) if the underlying gradient discretisation is coercive, consistent, limit-conforming, compact and piecewise constant, according to the following definitions, in which $\mathcal{D}$ denotes a gradient discretisation for homogeneous Dirichlet problems in the sense of Definition 3.1.

Definition 3.2 (Coercivity) Let

$$
C_{\mathcal{D}}=\max _{w \in X_{\mathcal{D}, 0} \backslash\{0\}} \frac{\left\|\Pi_{\mathcal{D}} w\right\|_{L^{2}(\Omega)}}{\|w\|_{\mathcal{D}}} .
$$


A sequence $\left(\mathcal{D}_{m}\right)_{m \in \mathbb{N}}$ of gradient discretisations for homogeneous Dirichlet problems is said to be coercive if there exists $C_{P} \in \mathbb{R}_{+}$such that $C_{\mathcal{D}_{m}} \leq C_{P}$ for all $m \in \mathbb{N}$.

Definition 3.3 (Consistency) Let $S_{\mathcal{D}}: H_{0}^{1}(\Omega) \rightarrow[0,+\infty)$ be defined by $\varphi \in H_{0}^{1}(\Omega) \mapsto S_{\mathcal{D}}(\varphi)$ with

$$
S_{\mathcal{D}}(\varphi)=\min _{w \in X_{\mathcal{D}, 0}}\left(\left\|\Pi_{\mathcal{D}} w-\varphi\right\|_{L^{2}(\Omega)}+\left\|\nabla_{\mathcal{D}} w-\nabla \varphi\right\|_{L^{2}(\Omega)^{d}}\right) .
$$

A sequence $\left(\mathcal{D}_{m}\right)_{m \in \mathbb{N}}$ of gradient discretisations for homogeneous Dirichlet problems is said to be consistent if

$$
\forall \varphi \in H_{0}^{1}(\Omega), \lim _{m \rightarrow \infty} S_{\mathcal{D}_{m}}(\varphi)=0
$$

Definition 3.4 (Limit-conformity) . Let

$$
H_{\mathrm{div}}(\Omega)=\left\{\boldsymbol{\varphi} \in L^{2}(\Omega)^{d}, \operatorname{div} \boldsymbol{\varphi} \in L^{2}(\Omega)\right\},
$$

and let $W_{\mathcal{D}}: H_{\mathrm{div}}(\Omega) \rightarrow[0,+\infty)$ be defined by $\boldsymbol{U} \mapsto W_{\mathcal{D}}(\boldsymbol{U})$, with

$$
W_{\mathcal{D}}(\boldsymbol{U})=\max _{w \in X_{\mathcal{D}, 0} \backslash\{0\}} \frac{1}{\|w\|_{\mathcal{D}}}\left|\int_{\Omega}\left(\nabla_{\mathcal{D}} w(\boldsymbol{x}) \cdot \boldsymbol{U}(\boldsymbol{x})+\Pi_{\mathcal{D}} w(\boldsymbol{x}) \operatorname{div} \boldsymbol{U}(\boldsymbol{x})\right) \mathrm{d} \boldsymbol{x}\right| .
$$

A sequence $\left(\mathcal{D}_{m}\right)_{m \in \mathbb{N}}$ of gradient discretisations for homogeneous Dirichlet problems is said to be limitconforming if

$$
\forall \boldsymbol{U} \in H_{\text {div }}(\Omega), \lim _{m \rightarrow \infty} W_{\mathcal{D}_{m}}(\boldsymbol{U})=0 .
$$

Definition 3.5 (Compactness) A sequence $\left(\mathcal{D}_{m}\right)_{m \in \mathbb{N}}$ of gradient discretisations for homogeneous Dirichlet problems is said to be compact if, for all sequence $u_{m} \in X_{\mathcal{D}_{m}, 0}$ such that $\left\|u_{m}\right\|_{\mathcal{D}_{m}}$ is bounded, the sequence $\left(\Pi_{\mathcal{D} m} u_{m}\right)_{m \in \mathbb{N}}$ is relatively compact in $L^{2}(\Omega)$.

Definition 3.6 (Piecewise constant function reconstruction) Let $\mathcal{D}$ be a gradient discretisation in the sense of Definition 3.1, and I be the finite set of the degrees of freedom, such that $X_{\mathcal{D}, 0}=\mathbb{R}^{I}$. We say that $\Pi_{\mathcal{D}}$ is a piecewise constant function reconstruction if there exists a family of open subsets of $\Omega$, denoted by $\left(\Omega_{i}\right)_{i \in I}$, such that $\bigcup_{i \in I} \overline{\Omega_{i}}=\bar{\Omega}, \Omega_{i} \cap \Omega_{j}=\emptyset$ for all $i \neq j$, and $\Pi_{\mathcal{D}} u=\sum_{i \in I} u_{i} \chi_{\Omega_{i}}$ for all $u=\left(u_{i}\right)_{i \in I} \in X_{\mathcal{D}, 0}$, where $\chi_{\Omega_{i}}$ is the characteristic function of $\Omega_{i}$.

Remark 3.1 Note that $\left\|\Pi_{\mathcal{D}} \cdot\right\|_{L^{2}(\Omega)}$ is not requested to be a norm on $X_{\mathcal{D}, 0}$. Indeed, in several schemes, some degrees of freedom are involved in the reconstruction of the gradient of the function, but not in that of the function itself. Hence it can occur that some of the $\Omega_{i}$ are empty.

\section{Definition 3.7 (Space-time discretisation)}

We say that $(\mathcal{D}, \delta t)$ is a space-time gradient discretisation of $\Omega \times] 0, T[$ if

- $\mathcal{D}=\left(X_{\mathcal{D}, 0}, \Pi_{\mathcal{D}}, \nabla_{\mathcal{D}}\right)$ is an approximate gradient discretisation of $\Omega$ in the sense of Definition 3.1,

- we have $t^{(0)}=0<t^{(1)} \ldots<t^{(N)}=T$, and we denote the discrete time step by $\delta t^{\left(n+\frac{1}{2}\right)}=$ $t^{(n+1)}-t^{(n)}$ for $n=0, \ldots, N-1$, and we let $\delta t=\left(\delta t^{\left(n+\frac{1}{2}\right)}\right)_{n=0, \ldots, N-1}$ and $|\delta t|=\max _{n=0, \ldots, N-1} \delta t^{\left(n+\frac{1}{2}\right)}$. 


\subsection{Definition of the scheme}

Let $(\mathcal{D}, \delta t)$ be a space-time discretisation of $\Omega \times] 0, T\left[\right.$ in the sense of Definition 3.7 such that $\Pi_{\mathcal{D}}$ is a piecewise constant function reconstruction in the sense of Definition 3.6. We define the following scheme for the discretisation of Problem (6)-(11) in the sense of Definition 1.1. For a given interpolation $u^{(0)}$ of $u_{\text {ini }}$, we seek $\left(u^{(n+1)}, p^{(n+1)}\right), n=0, \ldots, N-1$, such that, for any $w \in X_{\mathcal{D}, 0}$ and $n=0, \ldots, N-1$, we have:

$$
\begin{aligned}
& u^{(0)} \in X_{\mathcal{D}, 0}, u^{(n+1)} \in X_{\mathcal{D}, 0}, p^{(n+1)} \in X_{\mathcal{D}, 0}, \\
& \delta_{\mathcal{D}}^{\left(n+\frac{1}{2}\right)} u=\Pi_{\mathcal{D}} \frac{u^{(n+1)}-u^{(n)}}{\delta t^{\left(n+\frac{1}{2}\right)}}, \\
& \int_{\Omega}\left(\delta_{\mathcal{D}}^{\left(n+\frac{1}{2}\right)} u \Pi_{\mathcal{D}} w-\left(f_{1}\left(\Pi_{\mathcal{D}} u^{(n+1)}\right) \boldsymbol{v}_{\mathcal{D}}^{(n+1)}-\nabla_{\mathcal{D}} \psi\left(u^{(n+1)}\right)\right) \cdot \nabla_{\mathcal{D}} w\right) \mathrm{d} \boldsymbol{x} \\
& \quad=\int_{\Omega}\left(f_{1}(c) s^{+}-f_{1}\left(\Pi_{\mathcal{D}} u^{(n+1)}\right) s^{-}\right) \Pi_{\mathcal{D}} w \mathrm{~d} \boldsymbol{x}, \\
& -\int_{\Omega} \boldsymbol{v}_{\mathcal{D}}^{(n+1)} \cdot \nabla_{\mathcal{D}} w \mathrm{~d} \boldsymbol{x}=\int_{\Omega}\left(s^{+}-s^{-}\right) \Pi_{\mathcal{D}} w \mathrm{~d} \boldsymbol{x} \\
& \boldsymbol{v}_{\mathcal{D}}^{(n+1)}=-M\left(\Pi_{\mathcal{D}} u^{(n+1)}\right) \nabla_{\mathcal{D}} p^{(n+1)} .
\end{aligned}
$$

As in the case of the TP scheme, we use the notations $\Pi_{\mathcal{D}}, \nabla_{\mathcal{D}}$ and $\boldsymbol{v}_{\mathcal{D}}$ for the definition of spacetime dependent functions (see (24)). It is worth noticing that, in Scheme (27), the convection term is approximated by $f_{1}\left(\Pi_{\mathcal{D}} u^{(n+1)}\right) \boldsymbol{v}_{\mathcal{D}}^{(n+1)}$ and no upstream weighting. The following convergence proof can be extended to upstream weighting discretizations provided that a scheme dependent construction of the discrete Darcy fluxes is given (see [3] for the SUSHI scheme, and [5] for the Vertex Approximate Gradient scheme). We prove below the convergence of the scheme, but contrary to the TP scheme, no $L^{\infty}$ bound on $\Pi_{\mathcal{D}} u^{(n+1)}$ is known.

We finally introduce the function

$$
\Psi(s)=\int_{0}^{s} \psi(x) \mathrm{d} x, \forall s \in \mathbb{R},
$$

which is used several times in the convergence proof. We then have from the Assumption (12h),

$$
\Psi(s)=\int_{0}^{s}(\psi(x)-\psi(0)) \mathrm{d} x \leq L_{\psi} \int_{0}^{s} x \mathrm{~d} x=L_{\psi} \frac{s^{2}}{2},
$$

and, from Assumption (12i),

$$
\Psi(s) \geq \int_{0}^{s} \psi(x) \frac{\psi^{\prime}(x)}{L_{\psi}} \mathrm{d} x \geq \frac{\psi(s)^{2}}{2 L_{\psi}} \geq \frac{A^{2} s^{2}-2 B^{2}}{4 L_{\psi}} .
$$

Lemma 3.1 (discrete $L^{2}$ estimates) Under Hypotheses (12), let $(\mathcal{D}, \not t)$ be a space-time gradient discretisation of $\Omega \times] 0, T$ [ which is coercive in the sense of Definition 3.2 and such that $\Pi_{\mathcal{D}}$ is a piecewise constant function reconstruction in the sense of Definition 3.6. Then there exists $C_{1}>0$, only depending on $L_{\psi}, \bar{M}, \underline{M}, A, B, C_{P}>C_{\mathcal{D}}, C_{\mathrm{ini}}>\left\|u_{\mathrm{ini}}-\Pi_{\mathcal{D}} u^{(0)}\right\|_{L^{2}(\Omega)},\left\|s^{+}\right\|_{L^{2}(\Omega)},\left\|s^{-}\right\|_{L^{2}(\Omega)}$, such that, for any solution $(u, p)$ to this scheme,

$$
\left\|\Pi_{\mathcal{D}} u\right\|_{L^{2}(\Omega \times] 0, T[)} \leq C_{1},
$$




$$
\begin{gathered}
\left\|\boldsymbol{v}_{\mathcal{D}}\right\|_{L^{2}(\Omega \times] 0, T[)^{d}} \leq C_{1}, \\
\left\|\nabla_{\mathcal{D}} \psi(u)\right\|_{L^{2}(\Omega \times] 0, T[)^{d}} \leq C_{1} .
\end{gathered}
$$

As a consequence, there exists at least one solution $(u, p)$ to the scheme.

Proof. For a given time iteration $n>0$, we let $w=p^{(n+1)}$ in the second equation of (27) which leads to

$$
\int_{\Omega} M\left(\Pi_{\mathcal{D}} u^{(n+1)}\right)\left|\nabla_{\mathcal{D}} p^{(n+1)}\right|^{2} \mathrm{~d} \boldsymbol{x}=\int_{\Omega}\left(s^{+}-s^{-}\right) \Pi_{\mathcal{D}} p^{(n+1)} \mathrm{d} \boldsymbol{x} .
$$

Using hypothesis (12g)-(12d) and the Cauchy-Schwarz inequality, we have

$$
\underline{M}\left\|\nabla_{\mathcal{D}} p^{(n+1)}\right\|_{L^{2}(\Omega)}^{2} \leqslant\left(\left\|s^{-}\right\|_{L^{2}(\Omega)}+\left\|s^{+}\right\|_{L^{2}(\Omega)}\right)\left\|\Pi_{\mathcal{D}} p^{(n+1)}\right\|_{L^{2}(\Omega)} .
$$

Using (25) we have

$$
\left\|\nabla_{\mathcal{D}} p^{(n+1)}\right\|_{L^{2}(\Omega)} \leqslant C_{p}\left(\left\|s^{-}\right\|_{L^{2}(\Omega)}+\left\|s^{+}\right\|_{L^{2}(\Omega)}\right) \frac{1}{\underline{M}} .
$$

Finally using the definition of $\boldsymbol{v}_{\mathcal{D}}^{(n+1)}$ and $(12 \mathrm{~g})$ we have

$$
\left\|\boldsymbol{v}_{\mathcal{D}}^{(n+1)}\right\|_{L^{2}(\Omega)^{d}} \leqslant C_{p}\left(\left\|s^{-}\right\|_{L^{2}(\Omega)}+\left\|s^{+}\right\|_{L^{2}(\Omega)}\right) \frac{\bar{M}}{\underline{M}},
$$

which proves (32).

We then let $w=\psi\left(u^{(n+1)}\right)$ in the first equation of (27), which leads to

$$
\begin{aligned}
& \int_{\Omega} \delta_{\mathcal{D}}^{\left(n+\frac{1}{2}\right)} u \Pi_{\mathcal{D}}\left(\psi\left(u^{(n+1)}\right) \mathrm{d} \boldsymbol{x}+\left\|\nabla_{\mathcal{D}} \psi\left(u^{(n+1)}\right)\right\|_{L^{2}(\Omega)}^{2}\right. \\
= & \int_{\Omega}\left(f_{1}(c) s^{+}-f_{1}\left(\Pi_{\mathcal{D}} u^{(n+1)}\right) s^{-}\right) \Pi_{\mathcal{D}} \psi\left(u^{(n+1)}\right) \mathrm{d} \boldsymbol{x}+\int_{\Omega} f_{1}\left(\Pi_{\mathcal{D}} u^{(n+1)}\right) \boldsymbol{v}_{\mathcal{D}}^{(n+1)} \cdot \nabla_{\mathcal{D}} \psi\left(u^{(n+1)}\right) \mathrm{d} \boldsymbol{x} .
\end{aligned}
$$

Using twice the fact that $r_{1} r_{2} \leqslant \frac{1}{4} r_{1}^{2}+r_{2}^{2}$ which holds for any real numbers $r_{1}, r_{2}$ together with (25)-(12f) and the Cauchy-Schwarz inequality, we have

$$
\begin{aligned}
\int_{\Omega} \delta_{\mathcal{D}}^{\left(n+\frac{1}{2}\right)} u \Pi_{\mathcal{D}} \psi\left(u^{(n+1)}\right) \mathrm{d} \boldsymbol{x}+\frac{1}{2}\left\|\nabla_{\mathcal{D}} \psi\left(u^{(n+1)}\right)\right\|_{L^{2}(\Omega)}^{2} & \\
& \leqslant\left(C_{p}\left(\left\|s^{-}\right\|_{L^{2}(\Omega)}+\left\|s^{+}\right\|_{L^{2}(\Omega)}\right)+\left\|\boldsymbol{v}^{(n+1)}\right\|_{L^{2}(\Omega)^{d}}\right) .
\end{aligned}
$$

Using the function $\Psi$ defined by (28) and the equality

$$
\int_{r_{1}}^{r_{2}} \psi(x) \mathrm{d} x=\Psi\left(r_{2}\right)-\Psi\left(r_{1}\right)=\psi\left(r_{2}\right)\left(r_{2}-r_{1}\right)-\underbrace{\int_{r_{1}}^{r_{2}} \psi^{\prime}(x)\left(x-r_{1}\right) \mathrm{d} x}_{\geqslant 0},
$$

we obtain

$$
\begin{aligned}
\int_{\Omega} \frac{\Pi_{\mathcal{D}}\left(\Psi\left(u^{(n+1)}\right)-\Psi\left(u^{(n)}\right)\right)}{\delta t^{\left(n+\frac{1}{2}\right)}} \mathrm{d} \boldsymbol{x}+\frac{1}{2} \| \nabla_{\mathcal{D}} \psi & \left(u^{(n+1)}\right) \|_{L^{2}(\Omega)}^{2} \\
\leqslant & \underbrace{\left(C_{p}\left(\left\|s^{-}\right\|_{L^{2}(\Omega)}+\left\|s^{+}\right\|_{L^{2}(\Omega)}\right)+\left\|\boldsymbol{v}^{(n+1)}\right\|_{L^{2}(\Omega)^{d}}\right)}_{=C_{2} \geq 0} .
\end{aligned}
$$


We multiply by $\delta t^{\left(n+\frac{1}{2}\right)}$ and sum on $0, \ldots, N-1$, which gives

$$
\left\|\Pi_{\mathcal{D}} \Psi\left(u^{(N)}\right)\right\|_{L^{1}(\Omega)}+\frac{1}{2}\left\|\nabla_{\mathcal{D}} \psi(u)\right\|_{\left.L^{2}(\Omega \times] 0, T\right)^{d}}^{2} \leq C_{2} T+\left\|\Pi_{\mathcal{D}} \Psi\left(u^{(0)}\right)\right\|_{L^{1}(\Omega)},
$$

which in turn yields, thanks to (29) and (30),

$$
\frac{1}{2 L_{\psi}}\left\|\Pi_{\mathcal{D}} \psi\left(u^{(N)}\right)\right\|_{L^{2}(\Omega)}^{2}+\frac{1}{2}\left\|\nabla_{\mathcal{D}} \psi(u)\right\|_{L^{2}(\Omega \times] 0, T[)^{d}}^{2} \leq C_{2} T+\frac{L_{\psi}}{2}\left\|\Pi_{\mathcal{D}} u^{(0)}\right\|_{L^{2}(\Omega)}^{2} .
$$

This proves (33). Finally by using (25) and the hypothesis (12i) we obtain (31).

As in the case of the TP scheme, from these estimates, we get existence of a solution by a topological degree argument (see also [14]).

Lemma 3.2 (Estimate on the time translates) Under Hypotheses (12), let $(\mathcal{D}, \delta t)$ be a space-time gradient discretisation of $\Omega \times] 0, T$ [ which is coercive in the sense of Definition 3.2 and such that $\Pi_{\mathcal{D}}$ is a piecewise constant function reconstruction in the sense of Definition 3.6. Then there exists $C_{2}>0$, only depending on $L_{\psi}, \bar{M}, \underline{M}, A, B, C_{P}>C_{\mathcal{D}}, C_{\mathrm{ini}}>\left\|u_{\mathrm{ini}}-\Pi_{\mathcal{D}} u^{(0)}\right\|_{L^{2}(\Omega)},\left\|s^{+}\right\|_{L^{2}(\Omega)},\left\|s^{-}\right\|_{L^{2}(\Omega)}$, such that, for any solution $(u, p)$ to this scheme,

$$
\left.\left\|\Pi_{\mathcal{D}} \psi(u)(\cdot, \cdot+\tau)-\Pi_{\mathcal{D}} \psi(u)(\cdot, \cdot)\right\|_{L^{2}(\Omega \times(0, T-\tau))}^{2} \leq C_{2}(\tau+|\delta t|), \forall \tau \in\right] 0, T[.
$$

For the proof of this lemma we refer to [9, Lemma 2.3] where the term $\nabla_{\mathcal{D}} \zeta\left(u^{m}\right)$ in the proof of the mentioned paper is here replaced by $\nabla_{\mathcal{D}} \psi\left(u^{m}\right)-f_{1}\left(\Pi_{\mathcal{D}} u^{m}\right) \boldsymbol{v}_{\mathcal{D}}^{m}$ (this holds thanks to (12f)-(32)-(33)).

Lemma 3.3 (Compactness of approximate solution) Let Hypotheses (12) be fulfilled. Let $\left(\mathcal{D}_{m}, \delta t_{m}\right)_{m \in \mathbb{N}}$ be a sequence of space-time gradient discretisations, such that the associated sequence of approximate gradient approximations is limit-conforming (Definition 3.4) and compact (Definition 3.5, it is then coercive in the sense of Definition 3.2), and such that, for all $m \in \mathbb{N}, \Pi_{\mathcal{D} m}$ is a piecewise constant function reconstruction in the sense of Definition 3.6 and $\left|\delta t_{m}\right| \rightarrow 0$ as $m \rightarrow \infty$. For any $m \in \mathbb{N}$, let $u_{m}$ be a solution to Scheme (27), such that $\left\|u_{\mathrm{ini}}-\Pi_{\mathcal{D} m} u_{m}^{(0)}\right\|_{L^{2}(\Omega)} \rightarrow 0$ as $m \rightarrow \infty$. Then there exists $\bar{u} \in L^{2}(\Omega \times] 0, T[)$ and $\bar{p} \in L^{2}\left(0, T ; H_{0}^{1}(\Omega)\right)$ and a subsequence of space-time gradient discretisations again denoted $\left(\mathcal{D}_{m}, \delta t_{m}\right)_{m \in \mathbb{N}}$ such that, as $m \rightarrow \infty$,

1. $\psi\left(\Pi_{\mathcal{D} m} u_{m}\right)$ converges to $\psi(\bar{u}) \in L^{2}\left(0, T ; H_{0}^{1}(\Omega)\right)$ in $L^{2}(\Omega \times] 0, T[)$, (and therefore $\Pi_{\mathcal{D} m} u_{m}$ converges in $L^{2}(\Omega \times] 0, T[)$ to $\left.\bar{u}\right)$,

2. $\nabla_{\mathcal{D} m} \psi\left(u_{m}\right)$ converges to $\nabla \psi(\bar{u})$ weakly in $L^{2}(\Omega \times] 0, T[)^{d}$,

3. $\Pi_{\mathcal{D} m} p_{m}$ converges to $\bar{p} \in L^{2}\left(0, T ; H_{0}^{1}(\Omega)\right)$ weakly in $L^{2}(\Omega \times] 0, T[)$,

4. $\nabla_{\mathcal{D} m} p_{m}$ converges to $\nabla \bar{p}$ weakly in $L^{2}(\Omega \times] 0, T[)^{d}$.

PROOF. We list here the main ideas of the proof for each item.

1. We use Lemma 3.2 on the time translates, the compactness of $\left(\mathcal{D}_{m}\right)_{m \in \mathbb{N}}$ and the discrete AubinSimon Theorem as in [9]. The strong convergence of $\Pi_{\mathcal{D} m} u_{m}$ results from the fact that $\psi$ is strictly increasing,

2. Based on the previous point, the estimate (33) and the limit-conformity of $\left(\mathcal{D}_{m}\right)_{m \in \mathbb{N}}$. 
3. and 4. result from the estimate (32), the Hypothesis (12g), the coercivity and the limit-conformity of $\left(\mathcal{D}_{m}\right)_{m \in \mathbb{N}}$.

Lemma 3.4 (Convergence of approximate solution) Under the same hypotheses as that of Lemma 3.3, and moreover assuming the consistency of $\left(\mathcal{D}_{m}\right)_{m \in \mathbb{N}}$ in the sense of Definition 3.3, then the pair $(\bar{u}, \bar{p})$ (whose existence is given by Lemma 3.3) is a weak solution of the Problem (6)-(11) in the sense of Definition 1.1.

Proof. Let $m \in \mathbb{N}$, and let us denote $\mathcal{D}=\mathcal{D}_{m}$ (belonging to the above subsequence) and drop some indices $m$ for the simplicity of the notation. Let $g \in C_{c}^{\infty}([0, T))$ and $\varphi \in C_{c}^{\infty}(\Omega)$, and let $w \in X_{\mathcal{D}, 0}$ be such that

$$
w=\underset{z \in X_{\mathcal{D}, 0}}{\operatorname{argmin}} S_{\mathcal{D}}(\varphi)
$$

We first take as test function in the second equation of (27) the function $\delta t^{\left(n+\frac{1}{2}\right)} g\left(t^{(n)}\right) w$. Thanks to the strong convergence of $\Pi_{\mathcal{D} m} u_{m}$ to $\bar{u}$, to the weak convergence of $\nabla_{\mathcal{D} m} p_{m}$ to $\nabla \bar{p}$ and to the consistency of $\left(\mathcal{D}_{m}\right)_{m \in \mathbb{N}}$, we get that (14),(15) hold (we use the fact that the set

$$
\mathcal{T}=\left\{\sum_{i=1}^{q} g_{i}(t) \varphi_{i}(\boldsymbol{x}): q \in \mathbb{N}, g_{i} \in C_{c}^{\infty}[0, T), \varphi_{i} \in C_{c}^{\infty}(\Omega)\right\}
$$

is dense in $\left.C_{c}^{\infty}(\Omega \times[0, T))\right)$. We then take as test function in the first equation of (27) the function $\delta t^{\left(n+\frac{1}{2}\right)} g\left(t^{(n)}\right) w$, and we sum the resulting equation on $n=0, \ldots, N-1$. We get,

$$
T_{1}^{(m)}+T_{2}^{(m)}+T_{3}^{(m)}=T_{4}^{(m)},
$$

with

$$
\begin{gathered}
T_{1}^{(m)}=\sum_{n=0}^{N-1} \delta t^{\left(n+\frac{1}{2}\right)} g\left(t^{(n)}\right) \int_{\Omega} \delta_{\mathcal{D}}^{\left(n+\frac{1}{2}\right)} u(\boldsymbol{x}) \Pi_{\mathcal{D}} w(\boldsymbol{x}) \mathrm{d} \boldsymbol{x}, \\
T_{2}^{(m)}=\sum_{n=0}^{N-1} g\left(t^{(n)}\right) \int_{\Omega} f_{1}\left(\Pi_{\mathcal{D}} u^{(n+1)}(\boldsymbol{x})\right) M\left(\Pi_{\mathcal{D}} u^{(n+1)}(\boldsymbol{x})\right) \nabla_{\mathcal{D}} p^{(n+1)}(\boldsymbol{x}) \cdot \nabla_{\mathcal{D}} w(\boldsymbol{x}) \mathrm{d} \boldsymbol{x}, \\
T_{3}^{(m)}=\sum_{n=0}^{N-1} g\left(t^{(n)}\right) \int_{\Omega} \nabla_{\mathcal{D}} \psi\left(u^{(n+1)}\right)(\boldsymbol{x}) \cdot \nabla_{\mathcal{D}} w(\boldsymbol{x}) \mathrm{d} \boldsymbol{x},
\end{gathered}
$$

and

$$
T_{4}^{(m)}=\sum_{n=0}^{N-1} g\left(t^{(n)}\right) \int_{t^{(n)}}^{t^{(n+1)}} \int_{\Omega}\left(f_{1}(c(\boldsymbol{x})) s^{+}(\boldsymbol{x})-f_{1}\left(\Pi_{\mathcal{D}} u^{(n+1)}(\boldsymbol{x})\right) s^{-}(\boldsymbol{x})\right) \Pi_{\mathcal{D}} w(\boldsymbol{x}) \mathrm{d} \boldsymbol{x} \mathrm{d} t .
$$

Writing

$$
T_{1}^{(m)}=-\int_{0}^{T} g^{\prime}(t) \int_{\Omega} \Pi_{\mathcal{D}} u(\boldsymbol{x}, t) \Pi_{\mathcal{D}} w(\boldsymbol{x}) \mathrm{d} \boldsymbol{x} \mathrm{d} t-g(0) \int_{\Omega} \Pi_{\mathcal{D}} u^{(0)}(\boldsymbol{x}) \Pi_{\mathcal{D}} w(\boldsymbol{x}) \mathrm{d} \boldsymbol{x},
$$


we get, using Lemma 3.3 and the consistency of the discretisation, that

$$
\lim _{m \rightarrow \infty} T_{1}^{(m)}=-\int_{0}^{T} g^{\prime}(t) \int_{\Omega} \bar{u}(\boldsymbol{x}, t) \varphi(\boldsymbol{x}) \mathrm{d} \boldsymbol{x} \mathrm{d} t-g(0) \int_{\Omega} u_{\mathrm{ini}}(\boldsymbol{x}) \varphi(\boldsymbol{x}) \mathrm{d} \boldsymbol{x} .
$$

We also immediately get, using the convergence of $\Pi_{\mathcal{D} m} u_{m}$ to $\bar{u}$ in $L^{2}(\Omega \times] 0, T[)$, that

$$
\lim _{m \rightarrow \infty} T_{3}^{(m)}=\int_{0}^{T} g(t) \int_{\Omega} \nabla \psi(\bar{u}(\boldsymbol{x}, t)) \cdot \nabla \varphi(\boldsymbol{x}) \mathrm{d} \boldsymbol{x} \mathrm{d} t .
$$

and

$$
\lim _{m \rightarrow \infty} T_{4}^{(m)}=\int_{0}^{T} g(t) \int_{\Omega}\left(f_{1}(c(\boldsymbol{x})) s^{+}(\boldsymbol{x})-f_{1}(\bar{u}(\boldsymbol{x}, t)) s^{-}(\boldsymbol{x})\right) \varphi(\boldsymbol{x}) \mathrm{d} \boldsymbol{x} \mathrm{d} t .
$$

Similarly, we also have

$$
\lim _{m \rightarrow \infty} T_{2}^{(m)}=\int_{0}^{T} g(t) \int_{\Omega} f_{1}(\bar{u}(\boldsymbol{x}, t)) M(\bar{u}(\boldsymbol{x}, t)) \nabla \bar{p}(\boldsymbol{x}, t) \cdot \nabla \varphi(\boldsymbol{x}) \mathrm{d} \boldsymbol{x} \mathrm{d} t .
$$

Hence we get that (13) holds. Thanks again to the density in $C_{c}^{\infty}(\Omega \times[0, T))$ of the set $\mathcal{T}$ defined by (35), we conclude the proof that $(\bar{u}, \bar{p})$ is a weak solution of the Problem (6)-(11) in the sense of Definition 1.1.

Lemma 3.5 (Strong convergence of global pressure) Under the same hypotheses as that of Lemmas 3.3 and 3.4, which prove that $(\bar{u}, \bar{p})$ is a weak solution of the Problem (6)-(11) in the sense of Definition 1.1 , we have that, as $m \rightarrow \infty$,

1. $\Pi_{\mathcal{D} m} p_{m}$ converges to $\bar{p}$ strongly in $L^{2}(\Omega \times] 0, T[)$,

2. $\nabla_{\mathcal{D} m} p_{m}$ converges to $\nabla \bar{p}$ strongly in $L^{2}(\Omega \times] 0, T[)^{d}$.

PROOF. Let $m \in \mathbb{N}$, we denote $\mathcal{D}=\mathcal{D}_{m}$ (belonging to the suitable subsequence) and drop some indices $m$ for the simplicity of the notation. We then let $w=\Pi_{\mathcal{D}} p^{n+1}$ in the last two equations of (27), mutliply by $\delta t^{\left(n+\frac{1}{2}\right)}$ and sum the resulting equation on $n=0, \ldots, N-1$. We obtain

$\sum_{n=0}^{N-1} \delta t^{\left(n+\frac{1}{2}\right)} \int_{\Omega} M\left(\Pi_{\mathcal{D}} u^{(n+1)}(\boldsymbol{x})\right) \nabla_{\mathcal{D}} p^{(n+1)}(\boldsymbol{x}) \cdot \nabla_{\mathcal{D}} p^{(n+1)}(\boldsymbol{x}) \mathrm{d} \boldsymbol{x}=\sum_{n=0}^{N-1} \delta t^{\left(n+\frac{1}{2}\right)} \int_{\Omega}\left(s^{+}(\boldsymbol{x})-s^{-}(\boldsymbol{x})\right) \Pi_{\mathcal{D}} p^{(n+1)}(\boldsymbol{x}) \mathrm{d} \boldsymbol{x}$

This can be rewritten as follow

$$
\int_{0}^{T} \int_{\Omega} M\left(\Pi_{\mathcal{D}} u(\boldsymbol{x}, t)\right) \nabla_{\mathcal{D}} p(\boldsymbol{x}, t) \cdot \nabla_{\mathcal{D}} p(\boldsymbol{x}, t) \mathrm{d} \boldsymbol{x} \mathrm{d} t=\int_{0}^{T} \int_{\Omega}\left(s^{+}(\boldsymbol{x})-s^{-}(\boldsymbol{x})\right) \Pi_{\mathcal{D}} p(\boldsymbol{x}, t) \mathrm{d} \boldsymbol{x} \mathrm{d} t
$$

Using the weak convergence of $\Pi_{\mathcal{D}} p$ (Lemma 3.3), we have,

$$
\lim _{m \rightarrow \infty} \int_{0}^{T} \int_{\Omega}\left(s^{+}(\boldsymbol{x})-s^{-}(\boldsymbol{x})\right) \Pi_{\mathcal{D}} p(\boldsymbol{x}, t) \mathrm{d} \boldsymbol{x} \mathrm{d} t=\int_{0}^{T} \int_{\Omega}\left(s^{+}(\boldsymbol{x})-s^{-}(\boldsymbol{x})\right) \bar{p}(\boldsymbol{x}, t) \mathrm{d} \boldsymbol{x} \mathrm{d} t
$$

Moreover, since $(\bar{u}, \bar{p})$ is a weak solution (Lemma 3.4),

$$
\int_{0}^{T} \int_{\Omega} M(\bar{u}(\boldsymbol{x}, t)) \nabla \bar{p}(\boldsymbol{x}, t) \cdot \nabla \bar{p}(\boldsymbol{x}, t) \mathrm{d} \boldsymbol{x} \mathrm{d} t=\int_{0}^{T} \int_{\Omega}\left(s^{+}(\boldsymbol{x})-s^{-}(\boldsymbol{x})\right) \bar{p}(\boldsymbol{x}, t) \mathrm{d} \boldsymbol{x} \mathrm{d} t
$$


Thus,

$$
\lim _{m \rightarrow \infty} \int_{0}^{T} \int_{\Omega} M\left(\Pi_{\mathcal{D}} u(\boldsymbol{x}, t)\right) \nabla_{\mathcal{D}} p(\boldsymbol{x}, t) \cdot \nabla_{\mathcal{D}} p(\boldsymbol{x}, t) \mathrm{d} \boldsymbol{x} \mathrm{d} t=\int_{0}^{T} \int_{\Omega} M(\bar{u}(\boldsymbol{x}, t)) \nabla \bar{p}(\boldsymbol{x}, t) \cdot \nabla \bar{p}(\boldsymbol{x}, t) \mathrm{d} \boldsymbol{x} \mathrm{d} t
$$

On the other hand, we have

$$
\begin{aligned}
\int_{0}^{T} \int_{\Omega} M\left(\Pi_{\mathcal{D}} u(\boldsymbol{x}, t)\right) & \left(\nabla_{\mathcal{D}} p(\boldsymbol{x}, t)-\nabla \bar{p}(\boldsymbol{x}, t)\right) \cdot\left(\nabla_{\mathcal{D}} p(\boldsymbol{x}, t)-\nabla \bar{p}(\boldsymbol{x}, t)\right) \mathrm{d} \boldsymbol{x} \mathrm{d} t= \\
& \int_{0}^{T} \int_{\Omega} M\left(\Pi_{\mathcal{D}} u(\boldsymbol{x}, t)\right) \nabla_{\mathcal{D}} p(\boldsymbol{x}, t) \cdot \nabla_{\mathcal{D}} p(\boldsymbol{x}, t) \mathrm{d} \boldsymbol{x} \mathrm{d} t \\
& -2 \int_{0}^{T} \int_{\Omega} M\left(\Pi_{\mathcal{D}} u(\boldsymbol{x}, t)\right) \nabla_{\mathcal{D}} p^{(n)} \cdot \nabla \bar{p}(\boldsymbol{x}, t) \mathrm{d} \boldsymbol{x} \mathrm{d} t \\
& +\int_{0}^{T} \int_{\Omega} M\left(\Pi_{\mathcal{D}} u(\boldsymbol{x}, t)\right) \nabla \bar{p}(\boldsymbol{x}, t) \cdot \nabla \bar{p}(\boldsymbol{x}, t) \mathrm{d} \boldsymbol{x} \mathrm{d} t
\end{aligned}
$$

Then, by using the strong convergence of $\Pi_{\mathcal{D}} u$, the weak convergence of $\nabla_{\mathcal{D}} p$ and the hypothesis $(12 \mathrm{~g})$, we have,

$$
\lim _{m \rightarrow \infty} \underbrace{\int_{0}^{T} \int_{\Omega} M\left(\Pi_{\mathcal{D}} u(\boldsymbol{x}, t)\right)\left(\nabla_{\mathcal{D}} p(\boldsymbol{x}, t)-\nabla \bar{p}(\boldsymbol{x}, t)\right) \cdot\left(\nabla_{\mathcal{D}} p^{(n)}-\nabla \bar{p}(\boldsymbol{x}, t)\right) \mathrm{d} \boldsymbol{x} \mathrm{d} t}_{\geqslant \underline{M}\left\|\nabla_{\mathcal{D}} p-\nabla \bar{p}\right\|_{L^{2}(\Omega \times] 0, T[)^{d}}^{2} \geqslant 0}=0
$$

This prove the strong convergence of $\nabla_{\mathcal{D}} p$.

Let us now turn to the strong convergence of $\Pi_{\mathcal{D}} p$ to $\bar{p}$. For a.e. $t \in(0, T)$, let $\widehat{u}_{m}(t)$ be defined by

$$
\widehat{p}_{m}(t)=\underset{z \in X_{\mathcal{D}_{m}, 0}}{\operatorname{argmin}}\left(\left\|\Pi_{\mathcal{D}_{m}} z-\bar{p}(t)\right\|_{L^{2}(\Omega)}+\left\|\nabla_{\mathcal{D}_{m}} z-\nabla \bar{p}(t)\right\|_{L^{2}(\Omega)^{d}}\right) .
$$

By using that $0 \in X_{\mathcal{D}_{m}, 0}$, we have

$$
\left\|\Pi_{\mathcal{D}_{m}} \widehat{p}_{m}(t)-\bar{p}(t)\right\|_{L^{2}(\Omega)}+\left\|\nabla_{\mathcal{D}_{m}} \widehat{p}_{m}(t)-\nabla \bar{p}(t)\right\|_{L^{2}(\Omega)^{d}} \leq\|\bar{p}(t)\|_{L^{2}(\Omega)}+\|\nabla \bar{p}(t)\|_{L^{2}(\Omega)^{d}} .
$$

Thus, since $\bar{p} \in L^{2}\left(0, T ; H_{0}^{1}(\Omega)\right)$, we get by dominated convergence that

$$
\lim _{m \rightarrow \infty} \int_{0}^{T}\left\|\Pi_{\mathcal{D}_{m}} \widehat{p}_{m}(t)-\bar{p}(t)\right\|_{L^{2}(\Omega)}^{2} \mathrm{~d} t=0
$$

and

$$
\lim _{m \rightarrow \infty} \int_{0}^{T}\left\|\nabla_{\mathcal{D}_{m}} \widehat{p}_{m}(t)-\nabla \bar{p}(t)\right\|_{L^{2}(\Omega)^{d}}^{2} \mathrm{~d} t=0 .
$$

We then have, thanks to the coercivity hypothesis,

$$
\left\|\Pi_{\mathcal{D}_{m}}\left(p_{m}-\widehat{p}_{m}\right)\right\|_{L^{2}(\Omega \times(0, T))} \leq C_{P}\left\|\nabla_{\mathcal{D}_{m}}\left(p_{m}-\widehat{p}_{m}\right)\right\|_{L^{2}(\Omega \times(0, T))^{d}} .
$$


We thus get, thanks to the triangle inequality, that

$$
\left\|\Pi_{\mathcal{D}_{m}} p_{m}-\bar{p}\right\|_{L^{2}(\Omega \times(0, T))} \leq C_{P}\left\|\nabla_{\mathcal{D}_{m}}\left(p_{m}-\widehat{p}_{m}\right)\right\|_{L^{2}(\Omega \times(0, T))^{d}}+\left\|\Pi_{\mathcal{D}_{m}} \widehat{p}_{m}-\bar{p}\right\|_{L^{2}(\Omega \times(0, T))},
$$

which implies, thanks again to the triangle inequality,

$$
\begin{aligned}
\left\|\Pi_{\mathcal{D}_{m}} p_{m}-\bar{p}\right\|_{L^{2}(\Omega \times(0, T))} & \leq\left\|\Pi_{\mathcal{D}_{m}} \widehat{p}_{m}-\bar{p}\right\|_{L^{2}(\Omega \times(0, T))} \\
& +C_{P}\left(\left\|\nabla_{\mathcal{D}_{m}} p_{m}-\nabla \bar{p}\right\|_{L^{2}(\Omega \times(0, T))^{d}}+\left\|\nabla_{\mathcal{D}_{m}} \nabla_{\mathcal{D}_{m}} \widehat{p}_{m}-\nabla \bar{p}\right\|_{L^{2}(\Omega \times(0, T))^{d}} .\right.
\end{aligned}
$$

We then get

$$
\lim _{m \rightarrow \infty}\left\|\Pi_{\mathcal{D}_{m}} p_{m}-\bar{p}\right\|_{L^{2}(\Omega \times(0, T))}=0
$$

hence concluding the proof.

\section{Discussion}

The TP finite volume scheme and the approximate gradient scheme are two alternative ways of discretising a two phase flow problem in porous media. One of the main properties of the TP finite volume scheme is that the resulting discrete diffusion operator is of the form

$$
\sum_{L \in \mathcal{M}} \tau_{K L}\left(u_{K}-u_{L}\right)
$$

where the transmissivities $\tau_{K L}$ are positive and such that $\tau_{K L}=\tau_{L K}$. Thanks to this form, the monotony and natural $L^{\infty}$ bounds may be shown to hold. In the case of the two phase flow problem, this entails that the approximate saturation remains bounded by 0 and 1 . Moreover, it allows to get some stability estimates by multiplying the conservation equation by a function of the unknown which differs from the unknown involved in the second order term (for instance in Section 2, we multiplied (20) by $u_{K}$ while the unknown in the second order term is $\psi(u)$ ). This latter technique has been used in the study of several problems, as for instance for:

- the convergence of the discretisation of a nonlinear convection/degenerate diffusion equations [11],

- the convergence of the discretisation of a linear diffusion problem with singular right hand sides or initial data [8],

- the approximation of two-phase flow in porous media, with dissolution or heterogeneous capillary curves $[4,15,16]$

Note also that it is easy to implement an upstream weighting scheme for a convection term together with the TP scheme, which ensures more stability. The main drawback is that the admissible meshes in this case are restricted, especially in the $3 \mathrm{D}$ case, since we require the orthogonality condition for the TP scheme to be consistent. This has led to the development of Multi-Point Flux Approximation schemes [1], whose main drawback is the lack of robustness on too distorted meshes.

On the other hand, the generic framework of the gradient schemes includes a large number of recent methods which remain robust on general meshes, in the sense that it provides convergent approximations using common properties which are satisfied by these methods. The convergence proof which is 
done here is therefore valid for any scheme of this family (finite element, mixed finite element, mimetic method...). The main drawback of this analysis is the fact that the bounds $[0,1]$ on the discrete solution cannot be imposed in this general setting; moreover, in order to obtain an estimate, in general one can

only use as test function the one which appear in the second order terms, using the fact that $\int_{\Omega}\left|\nabla_{\mathcal{D}} \cdot\right|^{2}$ is a norm.

Ongoing research is active to obtain schemes that preserve the maximum principle. However it seems difficult to obtain a discrete diffusion term of the form (37) with positive transmissivities from a consistent gradient on a general mesh; even though it is possible in certain cases, for instance for piecewise linear finite element on triangular Delaunay meshes [17]. Nonlinear schemes have also been proposed [19,20]; however, the question of convergence of gradient schemes for the problems cited in the above list remains open on general grids.

\section{References}

[1] Aavatsmark, I., Barkve, T., Boe, O., Mannseth, T.: Discretization on unstructured grids for inhomogeneous, anisotropic media. part i: Derivation of the methods. SIAM Journal on Sc. Comp. 19, $1700-1716(1998)$

[2] Alt, H.W., Luckhaus, S.: Quasilinear elliptic-parabolic differential equations. Math. Z. 183(3), 311-341 (1983)

[3] Brenner, K.: Hybrid finite volume scheme for two-phase flow in porous media (2012). URL http: //hal.archives-ouVertes.fr/hal-00680686

[4] Brenner, K., Cances, C., Hilhorst, D.: A convergent finite volume scheme for two-phase flows in porous media with discontinuous capillary pressure field. Finite Volumes for Complex Applications VI Problems \& Perspectives pp. 185-193 (2011)

[5] Brenner, K., Masson, R.: Convergence of a Vertex Centred Discretization of Two-Phase Darcy flows on General Meshes (2012). URL http://hal.archives-ouvertes.fr/ hal-00755072

[6] Chavent, G., Jaffré, J.: Mathematical models and finite elements for reservoir simulation. Elsevier (1986)

[7] Droniou, J., Eymard, R., Gallouet, T., Herbin, R.: Gradient schemes: a generic framework for the discretisation of linear, nonlinear and nonlocal elliptic and parabolic equations, accepted for publication in M3AS. URL http://hal.archives-ouvertes.fr/hal-00751551

[8] Droniou, J., Gallouët, T., Herbin, R.: A finite volume scheme for a noncoercive elliptic equation with measure data. SIAM J. Numer. Anal. 41(6), 1997-2031 (electronic) (2003). DOI 10.1137/ S0036142902405205. URL http://dx.doi.org/10.1137/S0036142902405205

[9] Eymard, R., Féron, P., Gallouet, T., Herbin, R., Guichard, C.: Gradient schemes for the Stefan problem. URL http://hal.archives-ouvertes.fr/hal-00751555

[10] Eymard, R., Gallouët, T., Herbin, R.: Finite volume methods. In: P.G. Ciarlet, J.L. Lions (eds.) Techniques of Scientific Computing, Part III, Handbook of Numerical Analysis, VII, pp. 713-1020. North-Holland, Amsterdam (2000) 
[11] Eymard, R., Gallouët, T., Herbin, R., Michel, A.: Convergence of a finite volume scheme for nonlinear degenerate parabolic equations. Numer. Math. 92(1), 41-82 (2002)

[12] Eymard, R., Guichard, C., Herbin, R.: Small-stencil 3d schemes for diffusive flows in porous media. ESAIM: Mathematical Modelling and Numerical Analysis 46(02), 265-290 (2012)

[13] Eymard, R., Henry, G., Herbin, R., Hubert, F., Klöfkorn, R., Manzini, G.: 3d benchmark on discretization schemes for anisotropic diffusion problems on general grids. Finite Volumes for Complex Applications VI Problems \& Perspectives pp. 895-930 (2011)

[14] Eymard, R., Herbin, R., Latché, J.C.: Convergence analysis of a colocated finite volume scheme for the incompressible Navier-Stokes equations on general 2 or $3 \mathrm{~d}$ meshes. SIAM J. Numer. Anal. 45(1), 1-36 (2007)

[15] Eymard, R., Herbin, R., Michel, A.: Mathematical study of a petroleum-engineering scheme. M2AN Math. Model. Numer. Anal. 37(6), 937-972 (2003)

[16] Eymard, R., Schleper, V.: Study of a numerical scheme for miscible two-phase flow in porous media. URL http://hal.archives-ouvertes.fr/hal-00741425

[17] Gallouët, T., Herbin, R.: Convergence of linear finite elements for diffusion equations with measure data. Comptes Rendus Mathematique 338(1), 81-84 (2004)

[18] Herbin, R., Hubert, F., et al.: Benchmark on discretization schemes for anisotropic diffusion problems on general grids. Finite volumes for complex applications V pp. 659-692 (2008)

[19] Le Potier, C.: A nonlinear finite volume scheme satisfying maximum and minimum principles for diffusion operators. Int. J. Finite 6(2), 20 (2009)

[20] Le Potier, C., Mahamane, A.: A nonlinear correction and maximum principle for diffusion operators with hybrid schemes. CR Acad. Sci. Paris, Ser. I 350, 101-106 (2012)

[21] Michel, A.: A finite volume scheme for two-phase immiscible flow in porous media. SIAM J. Numer. Anal. 41(4), 1301-1317 (electronic) (2003). DOI 10.1137/S0036142900382739. URL http://dx.doi.org/10.1137/S0036142900382739 